\title{
Location of bifurcation curves of low codimension on piecewise smooth differential systems
}

\author{
Claudio A. Buzzi, $\quad$ Set Pérez-González, \\ Departamento de Matemática, IBILCE, UNESP, \\ 15054-000, São José do Rio Preto, SP \\ E-mail: buzzi@ibilce.unesp.br, setperez@mat.uab.cat,
}

\begin{abstract}
$\underline{\text { ABSTRACT }}$
In the study of dynamical systems, the piecewise differential systems have an special interest since modern engineering applications require the piecewise modeling of a wide range of problems in mechanics, power electronics, control theory or biology. They seem to be more accurate models for some realistic applications than the smooth systems. Even the linear case can reproduce much of the complex behavior observed in smooth nonlinear systems, like chaos.

A piecewise smooth system consists of a finite set of ordinary differential equations

$$
\dot{x}=f_{i}(x), x \in R_{i} \subset \mathbb{R}^{n},
$$

where the vector fields $f_{i}$ are smooth, defined on disjoint open regions $R_{i}$, and are smoothly extensible to the closure of $R_{i}$. Regions $R_{i}$ are separated by an $(n-1)$-dimensional set $\Sigma$ called the switching boundary, which consists of finitely many smooth manifolds intersecting transversely. The union of $\Sigma$ and all $R_{i}$ covers the whole state space $D \subseteq \mathbb{R}^{n}$.

As in the smooth case, the limit cycles of piecewise system are worthy of an special attention. Essentially, a limit cycle, $\gamma$, is a periodic orbit such that at least one trajectory of the vector field, different from $\gamma$, approaches $\gamma$ in positive or negative time. We pay special attention to the study of existence of periodic orbits for piecewise systems from the point of view of the bifurcation theory. That is, we will consider degenerated situations and after parameter variations we will look for the appearance of limit cycles. In particular, our interests reach the birth of limit cycles from singular points or the display of limit cycles from a homoclinic loop.

In [2], they are analyzed local and global bifurcations of codimension 2 of planar Filippov systems. In particular, the authors obtain the bifurcation diagram of system (1),

$$
Z_{\epsilon, \mu}(x, y)= \begin{cases}X_{\epsilon}(x, y)=\left(\begin{array}{c}
1 \\
-x+\epsilon x^{2}+x^{4}
\end{array}\right) & \text { if } y>0 \\
Y_{\mu}(x, y)=\left(\begin{array}{c}
-1 \\
-(x-\mu)
\end{array}\right) & \text { if } y<0 .\end{cases}
$$

In this case, there exists a bifurcation curve, $\eta$, in the parameter space, $(\epsilon, \mu)$, characterizing the existence of a semi-stable limit cycle. See Figure 1.

We adapt some techniques of [1] to piecewise dynamical systems. This paper introduce a way to approximate by algebraic curves the bifurcation curve of some phenomena of display of limit cycles. In this case, $\eta$ has an algebraic expression and we have exactly characterized it.
\end{abstract}

Theorem 1. Let $\eta$ be the bifurcation curve corresponding to the existence of a semi-stable limit cycle for system (1) where $\epsilon$ and $\mu$ are real numbers. Then

$$
\eta \equiv\left(\mu^{3}+\epsilon \mu-1\right)\left(144 \mu^{4}+24 \epsilon \mu^{2}+36 \mu+5 \epsilon^{2}\right)=0 .
$$




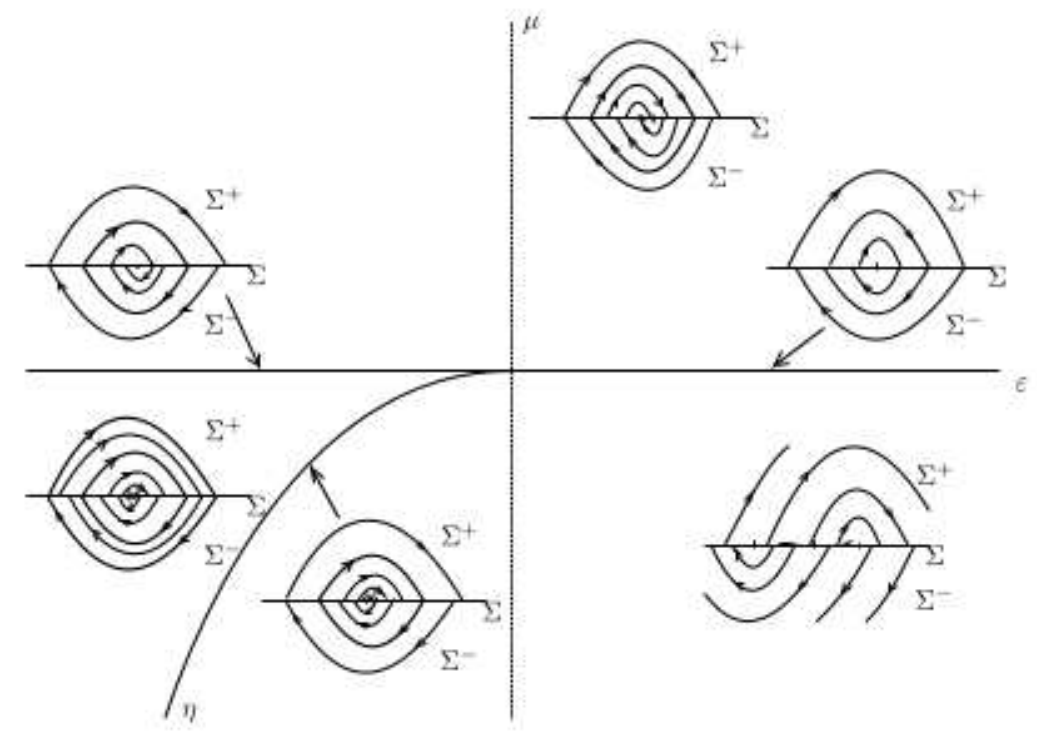

Figure 1: Bifurcation diagram of system (1) close to the origin, [2].

It is a work in progress and our purpose is to construct a method, as general as we could, to obtain results as the previous one.

Keywords: Piecewise smooth differential systems, bifurcation of limit cycles, location of limit cycles.

\section{References}

[1] A. Gasull, H. Giacomini and J. Torregrosa. Some results on homoclinic and heteroclinic connections in planar systems. Nonlinearity, 23 (2010), 2977-3001.

[2] M. Guardia, T. M. Seara and M. A. Teixeira. Generic bifurcations of low codimension of planar Filippov Systems. J. Differential Equations, 250 (2011), 1967-2023. 